\title{
Biomembranes Research Using Thermal and Cold Neutrons
}

\author{
F.A. Heberle ${ }^{\mathrm{a}, \mathrm{b}}$, D.A.A. Myles ${ }^{\mathrm{a}}$, and J. Katsaras ${ }^{\mathrm{a}-\mathrm{c}}$ \\ ${ }^{a}$ Biology and Soft Matter Division, Neutron Sciences Directorate, Oak Ridge, TN, 37831 \\ ${ }^{\mathrm{b}}$ Joint Institute for Neutron Sciences, Oak Ridge National Laboratory, Oak Ridge, TN, 37831 \\ ${ }^{c}$ Department of Physics and Astronomy, University of Tennessee, Knoxville, TN, 37996 \\ Corresponding author: katsarasj@ornl.gov
}




\begin{abstract}
In 1932 James Chadwick discovered the neutron using a polonium source and a beryllium target (Chadwick 1932). In a letter to Niels Bohr dated February 24, 1932, Chadwick wrote: "whatever the radiation from Be may be, it has most remarkable properties." Where it concerns hydrogenrich biological materials, the "most remarkable" property is the neutron's differential sensitivity for hydrogen and its isotope deuterium. Such differential sensitivity is unique to neutron scattering, which unlike X-ray scattering, arises from nuclear forces. Consequently, the coherent neutron scattering length can experience a dramatic change in magnitude and phase as a result of resonance scattering, imparting sensitivity to both light and heavy atoms, and in favorable cases to their isotopic variants. This article describes recent biomembranes research using a variety of neutron scattering techniques.
\end{abstract}




\section{Introduction}

Achieving a predictive understanding of cells requires moving beyond individual genes and proteins, toward functional, interacting systems compartmentalized in space and time. The various cellular compartments are defined by lipid bilayer-based membranes, composed of hundreds of distinct lipid species, and densely packed with a host of embedded and peripherally associated proteins. Far from being a passive barrier, membranes provide functional interfaces that mediate and control critical cellular processes, including fusion, trafficking, signaling and communication. A comprehensive understanding of cellular membranes - their structure, dynamic behavior and function - remains a grand challenge, primarily due to their intrinsically multi-molecular and mesoscopic scale. As two-dimensional fluid mixtures stabilized by weak interactions, membrane structure and function emerges only at a minimum length scale of approximately ten nanometers, corresponding to ensembles comprising hundreds of cooperating molecules. Furthermore, their inherent disorder makes membranes unsuitable for study by traditional structural biology methods such as crystallography that have revolutionized the study of proteins and nucleic acids (Strandberg, Dickerson et al. 2009; Garman 2014).

Among probes amenable to the study of membranes, neutrons stand out both for their nondestructive nature, and their multi-scale spatial and temporal information content. For example, neutron scattering techniques probe length scales ranging from angstroms to microns, dynamics occurring over picosecond to millisecond time scales, and kinetics spanning sub-seconds to hours. The value of neutrons in biology derives from the nature of their interaction with matter. Unlike X-rays, which interact with electrons, neutrons are scattered by atomic nuclei and can distinguish between elements of similar atomic and/or mass numbers. While X-ray interaction strength increases in proportion to atomic number, neutron scattering lengths show little variation across the periodic table (Table 1). Moreover, a distinct advantage of neutron scattering methods is their sensitivity to hydrogen, coupled with their ability to distinguish between the stable isotopes of hydrogen, protium and deuterium (i.e., ${ }^{1} \mathrm{H}$ and ${ }^{2} \mathrm{H}$, referred to subsequently as $\mathrm{H}$ and $\mathrm{D}$, respectively). Of the major classes of biomolecules (lipids, proteins, nucleic acids and carbohydrates), membrane lipids are the richest in hydrogen, and are thus readily detected and easily distinguished from other classes in a neutron scattering experiment. H/D isotopic labeling 
enhances selectivity and obviates the need for chemical tags and their associated artifacts (Morales-Penningston, $\mathrm{Wu}$ et al. 2010). As mentioned, thermal and cold neutrons are for the most part non-destructive, making them especially suitable for studying easily damaged unsaturated lipids, which are abundant in biology and the subject of much current interest. Important scattering techniques include small-angle neutron scattering (SANS), neutron diffraction (ND), neutron reflectometry (NR), neutron spin-echo spectroscopy (NSE) and quasielastic neutron scattering (QENS) (see Ankner, Heller et al. 2013 for introduction and review). Applications of these techniques range from probing the local environment of detergent solubilized proteins in single crystals, to solution scattering studies of membrane proteins incorporated in micelles, bicelles and vesicles, to scattering and diffraction from planar interfaces and surfaces in single layer or stacked lamellar systems.

Neutron scattering data alone do not provide a description of structures and events at the atomic level. However, simulations can often supply a critical link connecting scattering data to structure and dynamics, providing both interpretation and insights that guide subsequent experiments. In this regard, membrane systems have proven enormously challenging for simulation because of the number of atoms involved, the nature of their interactions, and the broad range of timescales relevant to biological membranes (nanoseconds to milliseconds). Molecular dynamics (MD) simulations have proven to be an effective approach for investigating these complex biological systems. In MD, the forces between atoms are described by a simplified empirical potential that attempts to mimic the 'real' underlying interaction potential. The primary output of an MD simulation is a trajectory of atomic coordinates through time, from which dynamic, structural and thermodynamic properties can be calculated. In favorable cases, MD simulations constitute a bridge between macroscopic (experimental) observations and microscopic details (Pan, Cheng et al. 2012; Pan, Cheng et al. 2014). This review article highlights current research where neutron scattering, in conjunction with other techniques including simulation, has played a significant role in addressing questions of current interest in molecular biology.

\section{Neutron scattering basics}




\subsection{Neutron Production}

Soon after the discovery of the neutron by Chadwick (Chadwick 1932), the first demonstrations of neutron diffraction were performed using a Rn-Be source (Halban and Preiswerk 1936; Mitchell and Powers 1936). The first neutron scattering experiments using nuclear reactors came later, using the CP-3 reactor at Argonne (Zinn 1947) and the X-10 pile at Oak Ridge (Wollan and Shull 1948). [For a historical review, see (Mason, Gawne et al. 2013).] These developments inspired the construction of facilities for the large-scale production of neutrons, subsequently creating new areas of research. Large quantities of neutrons suitable for scattering experiments are produced either by the fission of uranium-235 (e.g., ORNL's 85 MW High Flux Isotope Reactor HFIR) or by spallation, where high velocity particles (e.g., protons) produced by an accelerator impinge on a heavy metal target (liquid mercury in the case of ORNL's Spallation Neutron Source, SNS) (Harroun, Kucerka et al. 2009). The resulting fast neutrons are slowed ('thermalized') by passing through and interacting with a moderator (e.g., $\mathrm{H}_{2} \mathrm{O}, \mathrm{D}_{2} \mathrm{O}$, graphite, Be). They can be further slowed through interactions with a cold moderator, for example liquid hydrogen at $\sim 20 \mathrm{~K}$. Neutrons for scattering experiments generally have wavelengths ranging from 1-20 $\AA$, making them ideal probes for the study of a wide range of soft and hard materials.

\subsection{Neutron scattering techniques}

Neutron scattering is capable of probing material structural properties ranging from nanometers $\left(10^{-9} \mathrm{~m}\right)$ to micrometers $\left(10^{-6} \mathrm{~m}\right)$. Structure on these length scales is of interest to a wide range of scientific disciplines, including biology (Fitzsimmons, Bader et al. 2004; Shin, Choi et al. 2010; Breyton, Gabel et al. 2013; Mehan, Chinchalikar et al. 2013). As a general rule, the neutron wavelength $\lambda$ and range of detectable scattering angles $\left[\theta_{\min }, \theta_{\max }\right]$ determine the accessible experimental length scale $d$, through the relationship $d=\lambda / 2 \sin \theta$. A number of different scattering techniques, each with different geometries and sample requirements, have been developed to probe different structural aspects of a sample.

2.2.1 Small-angle neutron scattering. In a SANS experiment, the large-scale structure (10-10 $\AA$ ) of an unoriented sample (e.g., a protein in solution, or a vesicle suspension) is interrogated with longer wavelength neutrons produced by a cold moderator (liquid hydrogen or deuterium) and detected at small deflection angles (Fig. 1). For bilayer vesicles, the random sample 
orientation with respect to the incident beam results in the averaging of in-plane and out-of-plane structural information. It is often desirable to work at dilute concentrations to eliminate interparticle scattering contributions or 'structure factors', so that the observed scattering arises solely from scattering length density (SLD) variation within individual particles (the particle's 'form factor'), thereby simplifying data analysis. However, the use of dilute concentrations also reduces signal-to-background (and hence, spatial resolution) of a SANS experiment.

At the longer length scales probed by SANS, neutrons are differentially sensitive to the major classes of biological materials, enabling researchers to selectively highlight and examine the organization of individual components within a complex biological assembly. This is a critical capability: while analogous experiments using small-angle X-ray scattering (SAXS) can determine a complex's molecular envelope, SANS with contrast variation can in principle locate each of the individual components within the complex. For this reason, SANS is widely used for structural studies of lipid/protein and protein/DNA/RNA complexes, as well as highly dynamic or disordered complexes with flexible states (reviewed in Heller 2010). For example, SANS with contrast variation has been used to characterize the M. tuberculosis ParB-parS protein/DNA complex involved in plasmid-chromosome segregation and condensation (Chaudhuri, Gupta et al. 2011; Qian, Dean et al. 2012), to investigate the ezrin/PIP2/F-actin system from the membrane-cytoskeleton interface (Jayasundar, Ju et al. 2012), and to examine how variation in the length of the glutamine repeat of the huntingtin protein (associated with Huntington's disease) directs the structures undertaken by the polymerizing peptides along the aggregation pathway (Perevozchikova, Stanley et al. 2014).

2.2.2 Neutron diffraction. In contrast to powder SANS, neutron diffraction (ND) or crystallography experiments exploit the simple structure factor (a series of delta functions) that arises from highly ordered arrays of individual scatterers. In this way, the weak scattering of individual particles or bilayers is concentrated into discrete peaks, enhancing the signal in proportion to the multiplicity of the lattice and thereby improving signal-to-noise and structural resolution. While SANS typically requires indirect methods to obtain real-space information, in a diffraction experiment the real-space information is obtained directly from Fourier reconstruction of peak amplitudes, once the associated phase information is known. 
Neutron diffraction of single crystals is most frequently used to locate functionally important individual hydrogen atoms with near-atomic precision $(\sim 1.5-2.5 \AA)$ using crystals of biological molecules such as enzymes (see Golden and Vrielink 2014 for review). Less ordered components, such as detergents or lipids that may be associated with the protein, make little contribution to the high resolution diffraction data, and are difficult to locate in such structures. However, at lower resolutions $(\sim 10 \AA)$, ND is extremely sensitive to differences between the SLD of the protein and other components in single crystals, and can be combined with $\mathrm{H}_{2} \mathrm{O} / \mathrm{D}_{2} \mathrm{O}$ contrast variation techniques (discussed in section 2.3 below) to selectively highlight the distribution and nanoscale organization of lipids or detergents within single crystals of membrane proteins. With a new generation of single crystal instruments now entering operation around the world, including IMAGINE (Meilleur, Munshi et al. 2013) and MANDI (Schultz, Thiyagarajan et al. 2005) at ORNL, iBIX at J-PARC (Tanaka, Kusaka et al. 2010; Kusaka, Hosoya et al. 2013) and the BIODIFF instrument at FRM II (Munich), neutron analysis of membrane protein crystals is likely to expand significantly.

In a landmark study, neutron diffraction from positionally correlated lipid bilayers, in conjunction with site-specific deuteration, was used to determine the precise locations (to $\sim 1 \AA$ resolution) of labeled moieties within the bilayer (Buldt, Gally et al. 1978). Over the past 40 years, experiments of this type have determined the conformation of lipid molecules (Buldt, Gally et al. 1979; Zaccai, Buldt et al. 1979; Mihailescu, Vaswani et al. 2011), the location of biomolecules in model membranes (Worcester and Franks 1976; Kucerka, Marquardt et al. 2010), and the organization of cyanobacterial membranes (Liberton, Page et al. 2013). ND from oriented bilayer stacks offers the further advantage that in-plane and out-of-plane scattering can be isolated, either by changing the sample orientation with respect to the incident beam, or through the use of a two-dimensional detector to segregate transverse and lateral contributions along the detector's axes. However, a drawback of oriented bilayer stacks is that they are difficult to hydrate from water to $100 \%$ relative humidity, equivalent to samples in contact with bulk water (Katsaras 1997; Katsaras 1998). Nevertheless, even in partially dehydrated conditions, ND studies of oriented bilayers have provided meaningful contributions to the field of biomembranes (Han, Hristova et al. 2008; Deme, Cataye et al. 2014; Kent, Hunt et al. 2014) 
2.2.3 Neutron reflectometery. When biological interactions occur at planar surfaces, at interfaces, or in layered phases, neutron reflectometry (NR) using isotopic labeling and contrast variation can provide information about nanoscopic lipid-protein, lipid-peptide and lipid-lipid interactions (Krueger, Ankner et al. 1995; Fragneto, Graner et al. 2000; Krueger, Meuse et al. 2001; Burgess, Li et al. 2004; Doshi, Dattelbaum et al. 2005; Vacklin, Tiberg et al. 2005; Kent, Yim et al. 2008; Kent, Murton et al. 2010; Nanda, Datta et al. 2010). In an NR experiment, reflection of neutrons from thin films deposited on atomically flat surfaces, such as polished silicon or gold or at an airwater or liquid-liquid interface, gives detailed information on molecular organization along the axis perpendicular to the surface. In addition to deuteration of entire proteins and peptides to enhance contrast (Hellstrand, Grey et al. 2013), the distribution and organization of deuterated lipids can also be monitored in model membranes (Stidder, Fragneto et al. 2005), including lateral reorganization that can occur upon binding and multimerization of membrane associated proteins and complexes. Examples include the incorporation and distribution of cholesterol in model membranes (Stidder, Fragneto et al. 2005), and the voltage gating function of cation (e.g., $\mathrm{Na}+, \mathrm{K}+$ ) channel membrane proteins (Tronin, Nordgren et al. 2014).

\subsection{Contrast variation}

When it comes to biological materials, which are inherently rich in hydrogen, neutrons have a distinct advantage over many other experimental techniques. They are extremely sensitive to hydrogen, and scatter differently from biomolecules having different bulk hydrogen content such as proteins, lipids and nucleic acids (Jacrot 1976, Fig.2), thereby providing a natural contrast than can be used to distinguish the structure and dynamics of individual components within larger biological structures or assemblies. Moreover, hydrogen's ability to scatter neutrons, expressed as a scattering length, differs significantly from its deuterium isotope, not so much in magnitude as in phase: far from its resonance energy, the scattering length of $\mathrm{H}$ is negative, while that of D is positive ( $-3.74 \mathrm{fm}$ and $6.67 \mathrm{fm}$, respectively) (Sears 1992). It follows that dramatic changes in scattering amplitudes, and thus structural or dynamical information, can be achieved through the judicious substitution of $\mathrm{H}$ for D (Pabst, Kučerka et al. 2010). In biological materials this is achieved by varying the light $\left(\mathrm{H}_{2} \mathrm{O}\right)$ or heavy $\left(\mathrm{D}_{2} \mathrm{O}\right)$ water content of a sample, which enables tuning of the relative scattering power (or 'contrast') to reduce, enhance or match 
the scattering from individual components (Fig. 2). Furthermore, the scattering power and visibility of an individual component can be manipulated by varying its H/D composition using chemical or biological labeling techniques (Ankner, Heller et al. 2013). For example, deuterated vitamin $\mathrm{E}$ was used to determine its location in model membranes, which correlates well with the molecule's proposed antioxidant activities (Marquardt, Williams et al. 2013), while mixtures of protiated and deuterated lipids were used to detect lateral phase separation in lipid bilayers containing nanoscopic domains (Heberle, Doktorova et al. 2013; Heberle, Petruzielo et al. 2013; Pan, Heberle et al. 2013; Petruzielo, Heberle et al. 2013). Finally, because thermal neutrons are non-ionizing and therefore non-destructive, these contrast variation methods make it possible to examine the internal features of a sample in situ and under physiological conditions without damaging the sample.

\section{Neutron scattering from lipid bilayers}

Neutron scattering has made significant contributions to our understanding of lipid bilayer structure and organization. Neutrons and X-rays are sensitive to different parts of the lipid molecule and therefore give complementary information, which can be combined to obtain fully resolved structures of fluid bilayers. By introducing deuterated molecules into a protiated bilayer, it is possible to monitor the transverse location of the molecule within a bilayer. Similarly, through selective deuteration of a lipid species in a multicomponent mixture, it is possible to monitor lateral phase separation, including the formation of nanoscopic domains or "rafts" that cannot be resolved with conventional optical techniques. Analogous X-ray scattering experiments are difficult or impossible due to the inherently low sensitivity of X-rays to hydrogen, and their inability to distinguish between $\mathrm{H}$ and $\mathrm{D}$; for this reason, neutrons are uniquely suited to the study of rafts. We now discuss some specific examples of how neutron scattering techniques have been used to obtain structural information in lipid bilayers.

\subsection{Fluid bilayer structure.}

Biological membranes are thermally disordered, two-dimensional fluids, whose individual atomic positions are not well-localized as in a crystal, but rather are described by broad statistical averages. King and White (King and White 1986) were the first to propose a coarse-grained 
structure of a bilayer lipid, whereby neighboring atoms are grouped into quasimolecular fragments for the purpose of calculating scattering form factors for comparison to experimental scattering data. The functional forms describing these quasimolecular fragments (e.g., Gaussians or error functions) provide a fundamental link between the bilayer's matter distribution and various density profiles (e.g., electron density or neutron scattering length density), allowing different types of scattering and volumetric data to be combined in a single analysis, thereby increasing the information content and resolution of the structure. A fully resolved fluid bilayer structure comprises the distributions of a handful of quasimolecular fragments, typically $2-3$ headgroup components and 3-4 hydrocarbon components. Through the joint refinement of neutron and X-ray diffraction data, Wiener and White determined the fully resolved structure of a partially dehydrated fluid DOPC bilayer (Wiener, King et al. 1991; Wiener and White 1991; Wiener and White 1991; Wiener and White 1992; Wiener and White 1992).

Kucerka et al. extended the usefulness of this approach by introducing the Scattering Density Profile (SDP) analysis, which leverages the atomistic detail provided by MD simulations to guide the parsing of the lipid into quasimolecular fragments, in order to maximize the model's compatibility with X-ray and neutron experiments of differing contrast (Kucerka, Nagle et al. 2008). By combining SAXS data with SANS data at several $\mathrm{D}_{2} \mathrm{O} / \mathrm{H}_{2} \mathrm{O}$ ratios, the authors obtained the first fully resolved bilayer structure from a fully hydrated vesicle suspension. The SDP approach has since been used to solve the structures of biologically relevant lipids in fully hydrated fluid bilayers, including phosphatidylcholine (Kucerka, Gallova et al. 2009; Kucerka, Nieh et al. 2011), phosphatidylglycerol (Pan, Marquardt et al. 2014), phosphatidylserine (Pan, Cheng et al. 2014), phosphatidylethanolamine (Kucerka, van Oosten et al. 2015), and cardiolipin (Pan, Cheng et al. 2015). A key achievement of the SDP model is the robust determination of lipid areas and bilayer thicknesses that are crucial for the validation of MD force fields (reviewed in Heberle, Pan et al. 2012). Recently, Heftberger and coworkers extended the SDP analysis to multilamellar vesicles, enabling the determination of bending fluctuations and interbilayer interactions (Heftberger, Kollmitzer et al. 2014).

\subsection{Transverse location of bilayer components.}


Vitamin E was discovered in 1922 by Evans and Bishop (Evans and Bishop 1922), isolated from various oils (e.g., wheat germ, corn and cotton seed) by Emerson et al. in 1936 (Emerson, Emerson et al. 1936; Evans, Emerson et al. 1936), and synthesized by Karrer et al. in 1938 (Karrer, Fritzsche et al. 1938; Karrer, Fritzsche et al. 1938). It has since become clear that vitamin E comprises two families of compounds, namely tocopherols and tocotrienols, which differ in the number of double bonds in their side chains (Atkinson, Epand et al. 2008). Though tocopherols are widely used as antioxidants in the cosmetic and food industries, in vivo studies have shown that $\alpha$-tocopherol at physiological concentrations is unable to prevent oxidative damage (Azzi 2007).

Marquardt et al. (Marquardt, Williams et al. 2013) demonstrated that $\alpha$-tocopherol's location in different model membranes supports its role as an antioxidant (Fig. 3). Using selectively deuterated $\alpha$-tocopherol and a combination of techniques, including nuclear magnetic resonance, UV spectroscopy, and neutron diffraction, these authors found that $\alpha$-tocopherol manifests its antioxidant capabilities at the membrane's surface, either by intercepting reactive oxygen species entering the membrane, or by terminating lipid radicals formed in the membrane's interior. The latter is accomplished by virtue of the high conformational disorder possessed by polyunsaturated fatty acid chains, which 'snorkel' to the level of $\alpha$-tocopherol's hydroxyl group. Marquardt et al. concluded that $\alpha$-tocopherol is positioned such that its hydroxyl group, residing at the lipid/water interface, is easily accessed by ascorbate, which recycles the tocopheroxyl radical to $\alpha$-tocopherol. Importantly, it is thought that $\alpha$-tocopherol terminates lipid peroxide chain reactions in the membrane's interior.

\subsection{Membrane nanodomains.}

The previous two decades have witnessed a dramatic change in our understanding of cell membrane functionality, particularly as it concerns the lateral organization of lipids and proteins. It is now widely believed that membrane nanodomains, colloquially referred to as 'lipid rafts', play an active role in many cellular processes (Jacobson, Mouritsen et al. 2007; Lingwood and Simons 2010). Rafts are thought to be small (10-100 nm), heterogeneous and highly dynamic structures, rich in sterols and sphingolipids. Through the selective partitioning of membrane proteins between rafts and their surroundings, rafts can control protein-protein interactions, 
enhancing certain associations while suppressing others. In this manner, rafts are thought to mediate a range of cellular processes, including signal transduction (Holowka, Gosse et al. 2005), apoptosis (Gajate, Gonzalez-Camacho et al. 2009), cell adhesion and migration (Murai 2012), synaptic transmission (Sebastiao, Colino-Oliveira et al. 2013), cytoskeletal organization (Head, Patel et al. 2014), and protein sorting (Jacobson, Mouritsen et al. 2007), and have been implicated in HIV assembly on the inner leaflet of infected cells (Ono 2010). Rafts have been extensively studied in animal cells, and recent research has shown that they are also central to the organization and function of membranes in plants (Gerbeau-Pissot, Der et al. 2014) and microbes (reviewed in Bramkamp and Lopez 2015). The emerging consensus is that rafts are small (below the resolution limit of optical microscopy), chemically heterogeneous, and highly dynamic structures. The size, lifetime, and connectivity of rafts are crucial parameters for understanding their function.

Using SANS and acyl chain perdeuterated phospholipids, Heberle et al. (Heberle, Petruzielo et al. 2013) measured the composition dependence of membrane domain size in unilamellar vesicles (ULVs) in the absence of extrinsic probes. A clear dependence between domain size and bilayer thickness was established using SANS and $60 \mathrm{~nm}$ diameter ULVs composed of fixed proportions of cholesterol and 1,2-distearoyl-sn-glycero-3-phosphocholine (DSPC), mixed with varying amounts of 1-palmitoyl-2-oleoyl-sn-glycero-3-phosphocholine (POPC) and 1,2-dioleoyl$s n$-glycero-3-phosphocholine (DOPC). Specifically, domain size and bilayer thickness mismatch between coexisting liquid-ordered (Lo) and liquid-disordered (Ld) phases increased as a function of an increasing mole fraction of the non-biologically relevant DOPC lipid and a decreasing mole fraction of the biologically relevant POPC lipid [i.e., DOPC/ (DOPC + POPC)] (Fig. 4). The results suggest possible mechanisms in biological systems for regulating domain size through changes in lipid composition.

The study by Heberle et al. (Heberle, Petruzielo et al. 2013) described the detection of nanoscopic domains as small as $\sim 7 \mathrm{~nm}$ in radius in ULVs, using selective lipid deuteration to provide lateral contrast and Monte Carlo simulations to fit the SANS data (Fig. 4). Armstrong et al. (Armstrong, Marquardt et al. 2013) took a different approach to study the presence of nanoscopic Lo domains in membranes composed of 1,2-dipalmitoyl-sn-glycero-3- 
phosphocholine (DPPC) and $32.5 \mathrm{~mol} \%$ cholesterol. Using membranes aligned on silicon substrates, these authors exploited the neutron beam's coherence length (Armstrong, Barrett et al. 2012) to detect Lo domains (Fig. 5). Because the coherence length of X-rays and neutrons is such that only coherent spatially averaged structures are observed, nanoscopic domains are not easily detected. However, by tailoring the coherence length of the probe to the size of the feature in question, one can determine the size of nanoscopic domains. It should be pointed out, however, that relating domain size to the coherence length of the neutron beam is a nontrivial matter. As such, computer simulations are needed to calculate the in-plane structure factors for the difference coherence length neutron beams, which are then compared to experimental data (Armstrong, Barrett et al. 2012; Armstrong, Marquardt et al. 2013).

\section{Neutron scattering from membrane proteins and assemblies}

Understanding the interactions and function of membrane proteins within the fluid lipid environment of plasma membranes is a significant challenge - and critical need - in biology. Membrane proteins are crucial components of all higher forms of life, performing multiple functions within and between cells, including transmembrane transport of ions, electrons and metabolites, and in cell signaling, recognition and adhesion. More than $30 \%$ of mammalian genes encode membrane proteins, and membrane proteins constitute more than $60 \%$ of current drug targets. Membrane proteins are associated either with the polar headgroups at the surface of the lipid bilayer (peripheral membrane proteins) or embedded within the hydrophobic matrix (integral membrane proteins), and are now thought to organize and assemble into multiple types of compositionally distinct lipid domains (Lingwood and Simons 2010; Simons and Gerl 2010). Despite the critical importance of membrane proteins, their incorporation with the fluid plasma membrane complicates and restricts analysis using conventional high throughput techniques such as X-ray crystallography and NMR. Consequently, our understanding of the structure and function of membrane proteins in their native environment is still in its infancy.

The sensitivity of neutron diffraction and scattering to the hydrogen content of different biological materials makes these techniques important tools for studying the composition and interactions of protein-lipid complexes and assemblies (Ankner, Heller et al. 2013). The marked 
difference in SLD between natural lipids (which are contrast matched at $\sim 10-14 \% \mathrm{D}_{2} \mathrm{O}$ ) and proteins (which are contrast matched at $\sim 41 \% \mathrm{D}_{2} \mathrm{O}$ ) enables neutron contrast variation techniques to selectively highlight and examine the structure, function and dynamical properties of membrane proteins in biomimetic conditions. Moreover, the relative scattering and contrast between the lipid and protein components of such systems can be further manipulated and enhanced by the use of selective H/D labeling approaches, allowing the structure and dynamics of individual H/D-labeled components to be distinguished and analyzed within complex mutlicomponent systems and in lipid-associated states (Heinrich and Losche 2014). Advances in neutron instrumentation and labeling technologies now enable new classes of study of membrane protein-lipid assemblies in single crystals, in solution, and at planar interfaces and surfaces. Such information can be difficult — if not impossible - to obtain using other techniques. We now discuss some specific applications of neutron scattering techniques to the study of membrane/protein systems.

\subsection{Lipid/protein assemblies.}

The natural SLD difference between lipids and proteins makes SANS especially powerful for structural studies of membrane proteins in detergent-solubilized and lipid-associated states (Table 1). In its simplest use, SANS can determine the low resolution structure and conformation of detergent solubilized membrane proteins in solution, or more elegantly, to investigate protein structure, oligomerization and interactions when reconstituted into lipid carriers and biomimetic assemblies such as bicelles and nanodiscs (Midtgaard, Pedersen et al. 2014). Careful contrast matching then allows scattering from the protein and surrounding detergent or lipid molecules to be measured and distinguished, and for composite models of the protein, lipid and/or detergent components to be determined. For example, Penel et al. solved the detergent structure in single crystals of the OmpF porin from E. coli, showing how the detergent is structured and ordered around the membrane-spanning hydrophobic surface of the proteins (Penel, Pebay-Peyroula et al. 1998). In another example, Johs et al. used SANS to determine the ab initio structure of the large (550kDa) ApoB-100 protein component of low density lipoprotein (LDL), using measurements at the $18 \% \mathrm{D}_{2} \mathrm{O}$ contrast match point of the NonidetP40 detergent (Johs, Hammel et al. 2006). 
The power of contrast matching is best exploited when combined with selective H/D labeling approaches that enable individual components to be located within larger multi-protein complexes and assemblies. This is exemplified by an elegant neutron scattering analysis of the interaction of the bacterial toxin colicin $\mathrm{N}(\mathrm{ColN})$ with its outer membrane receptor protein OmpF (Clifton, Johnson et al. 2012) The authors used neutron reflectometry combined with selective deuteration to follow OmpF-dependent insertion of ColN in a DPPG monolayer, and used SANS to solve the translocon structure in detergent solution, removing the detergent scattering contribution through contrast matching. The study proposed a new structural model of the ColN-OmpF complex that provides functional insights for membrane translocation.

Lamellar diffraction of neutrons from stacked membrane bilayers has been widely used to analyze the organization and assembly of peptides and lipids in model membrane systems (Nagle and Tristram-Nagle 2000). In a recent, powerful example of this approach, Mihailescu et al. used neutron diffraction from stacked lamellar samples to investigate the interaction of a voltage sensor toxin (VSTx1) and a model lipid membrane (Mihailescu, Krepkiy et al. 2014). Using deuterated and hydrogenated VSTx1, the authors demonstrated the localization of the toxin at the headgroup region, which resulted in a thinning of the bilayer, as well as a toxin orientation within the membrane that facilitates formation of the toxin-channel complexes.

Recent advances in the development of tethered bilayers on gold (Krueger, Meuse et al. 2001) or silicon surfaces (Hughes, Howse et al. 2008), or on nanostructured substrates (Hertrich, Stetter et al. 2014), have enabled closer control of bilayer architecture and asymmetry that may better mimic the cellular environment and open the way to new classes of experiments. The development of magnetic contrast neutron reflectometry (MCNR), which uses a membrane supported on a magnetic substrate, and a magnetic field to modulate the SLD for spin polarized neutrons, has significantly improved the resolution and precision of the technique, allowing analysis of more complex lipid membrane systems (Le Brun, Holt et al. 2008; Holt, Le Brun et al. 2009). In an exciting recent application, Shen et al. demonstrated that MCNR provided structural resolution down to $1 \AA$ in their studies of the membrane translocation and assembly module (TAM), enabling the accurate measurement of protein domains projecting from the membrane layer (Shen, Leyton et al. 2014). 


\subsection{Complex assemblies: Structure and organization of photosynthetic systems}

Understanding the mechanisms that biological systems use to program the self-assembly (and disassembly) of interacting complexes and higher order assemblies that are dynamic, responsive, self-replicating and adaptive to environmental pressures is of key interest in biology and nanotechnologies. Neutron scattering is especially well-suited to analysis of the structure $\left(10-10^{4} \mathrm{~A}\right)$ and kinetics (seconds to hours) of such hierarchical systems, and has been used to characterize and understand how the assembly, packing fraction, distribution, organizational structure and pigment composition of natural antenna systems affects the efficiency and response of light energy conversion in plant photosynthetic machinery.

Neutron scattering approaches have allowed the detergent structure in single crystals of the photosynthetic reaction center to be determined (Roth, Lewitbentley et al. 1989), showing how the detergent is structured and ordered around the protein's membrane spanning hydrophobic surface. In studies of the integral membrane light-harvesting complex LH2, Prince et al. used H and D tail- and head-labeled detergents to show that the detergent tails are localized on the inner and outer hydrophobic surfaces of the protein (Prince, Howard et al. 2003, Fig. 6). In a separate study, Cardoso et al. used SANS measurements of $\mathrm{LH} 2$ at $15 \% \mathrm{D}_{2} \mathrm{O}$ (the contrast match point of the $\mathrm{OG}$ detergent) to show that the protein structure in solution is trimeric, and used measurements at $100 \% \mathrm{D}_{2} \mathrm{O}$ to accentuate and model the monolayer of detergent that surrounds the protein (Cardoso, Smolensky et al. 2009).

Fundamental studies of photosynthetic systems include investigating the solution structure of isolated cyanobacterial photosystem I in complex with detergent using SANS and MD simulation (Le, Harris et al. 2014), and the structure of chlorosomes in solution (Tang, Urban et al. 2010; Tang, Zhu et al. 2011; Tang and Blankenship 2012). In addition, whole cell studies of cyanobacterial cells were performed using SANS and revealed light dependent reorganization of the thylakoid membranes in response to light stimulus, giving new insights into the photosynthetic process (Liberton, Collins et al. 2013; Liberton, Page et al. 2013). In particular, the multi-membrane, supramolecular organization of these photosynthetic pathways makes it difficult to obtain information concerning the precise organization of the individual complexes. 
Research on applied photosynthesis has focused on developing approaches to entrap and stabilize chlorosomes (O'Dell, Beatty et al. 2012) within silica structures and the interactions of light harvesting antennas with synthetic block copolymers (Cardoso, Smolensky et al. 2011, Fig. 7).

\subsection{Complex assemblies: Hierarchical structure of virus particles}

Cell membrane structure and function are shaped and controlled through multiple protein-lipid interactions. At the molecular scale, protein-lipid interactions modulate and control local membrane physical properties, such as bilayer asymmetry, curvature (negative and positive) and lateral pressure, and exert dynamic control of local membrane composition, morphology and architecture. At larger scales, protein networks and scaffolds organize and assemble at the bilayer to separate and fuse membrane structures into sheets, tubes and vesicles that form partitions and connections within and between cells. Neutron scattering is sensitive across these multiple length scales, and since it is non-destructive, can provide information that spans from the dynamic assembly of protein-lipid complexes in membranes to the re-organization and control of hierarchical structures in viruses and in living cells.

As an example, SANS has been used to analyze the structure and assembly of the red clover necrotic mosaic virus in solution (Martin, He et al. 2013), and to characterize the structural differences in Sindbis virus particles that were produced from vertebrate and invertebrate cells (He, Piper et al. 2010; He, Piper et al. 2012). Sindbis is an envelope virus with a complex coreshell structure: the viral RNA is contained within an inner protein shell, itself surrounded by a membrane bilayer and finally an outer glycoprotein coat. The membrane bilayer is derived from the host in which the virus is produced, and the insect or vertebrate host-derived membrane can have different compositions and yet retain infectious activity between host species (Burge and Huang 1970; Knight, Schultz et al. 2009). SANS analysis showed significant structural differences between Sindbis virus particles grown in the different hosts, showing that the outer protein coat was more extended in virus grown in mammalian cells, and that while the radial position of the lipid bilayer did not change significantly, it contained significantly more cholesterol when grown in mammalian cells. 


\section{Concluding remarks}

Understanding the variety and complexity of membrane lipid-lipid and lipid-protein interactions in the plasma membrane will be essential to achieving an accurate model of lipid-mediated cellular function. The sensitivity of neutrons to hydrogen and deuterium enables the structurefunction relationship of biological systems to be examined across an extended range of length and time scales, and multiple levels of organization. This has inspired the development of a new generation of neutron scattering and imaging facilities (e.g., SNS, Oak Ridge, TN) that provide new and more sophisticated ways to tackle complex problems in biology. When combined with selective H/D-labeling, neutron scattering techniques offer possibilities to resolve outstanding biological problems.

\section{Acknowledgements}

FH, DAAM and JK are supported through the Scientific User Facilities Division of the DOE Office of Basic Energy Sciences (BES), under contract no. DE-AC05 00OR2275. 


\section{Tables}

Table I. Neutron coherent scattering lengths and incoherent cross-sections in biological materials.

\begin{tabular}{|c|c|c|c|}
\hline Isotope & $\begin{array}{l}\text { Atomic } \\
\text { Number }\end{array}$ & $\begin{array}{l}\text { S Neutron incoherent } \\
\text { cross section (Barns) }\end{array}$ & $\begin{array}{l}\text { Neutron coherent } \\
\text { scattering length } \\
(10-12 \mathrm{~cm})\end{array}$ \\
\hline $1 \mathrm{H}$ & 1 & 80.27 & -0.374 \\
\hline $2 \mathrm{H}(\mathrm{D})$ & 1 & 2.05 & 0.667 \\
\hline $12 \mathrm{C}$ & 6 & 0.00 & 0.665 \\
\hline $14 \mathrm{~N}$ & 7 & 0.49 & 0.937 \\
\hline 160 & 8 & 0.00 & 0.580 \\
\hline $24 \mathrm{Mg}$ & 12 & 0.00 & 0.549 \\
\hline $32 \mathrm{~S}$ & 16 & 0.00 & 0.280 \\
\hline
\end{tabular}




\section{Figures}

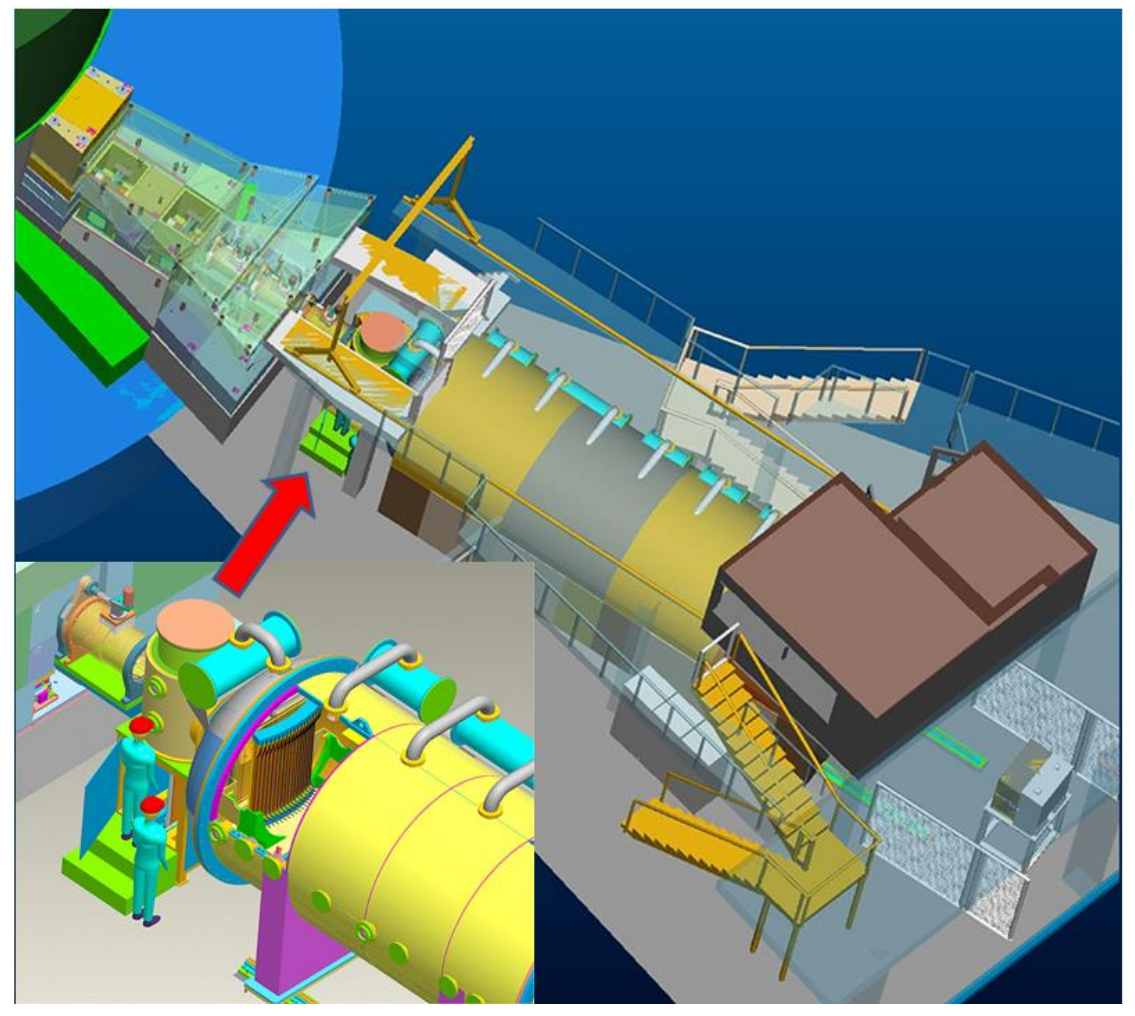

Figure 1 Schematic of the Extended Q-Range Small Angle Neutron Scattering (EQ-SANS) instrument at the Spallation Neutron Source (SNS, ORNL). The instrument is capable of interrogating samples ranging from 0.1 to $100 \mathrm{~nm}$. The two-dimensional detector is $1 \mathrm{~m}^{2}$ and the sample to detector distance can be varied, in the evacuated detector tank (green with gray stripe), from 1.3 to $9 \mathrm{~m}$. The inset shows the sample chamber, which is located at one end of the detector tank. Neutrons are produced at the mercury target and transported to sample chamber, located 14 $\mathrm{m}$ from the target. 


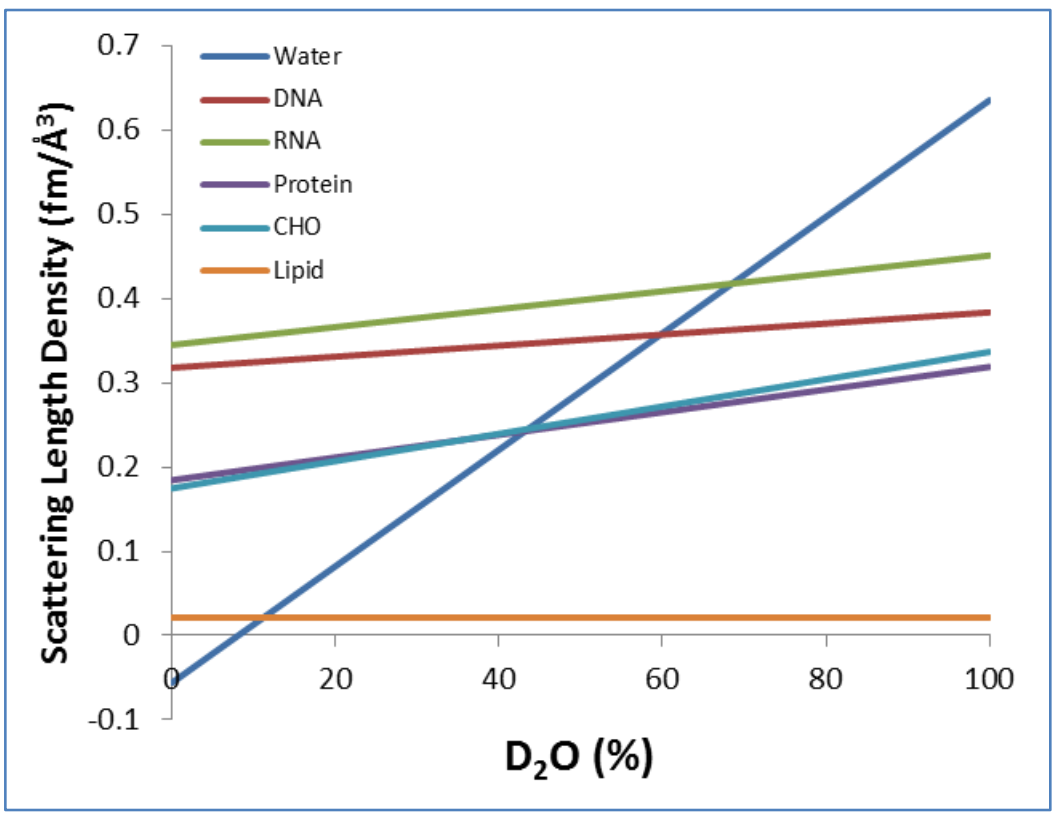

Figure 2 Neutron contrast variation. Plots showing neutron scattering length density (SLD) as a function of H20/D20 content for different class biomolecules. Match points (i.e., no neutron contrast) occur when the SLD for the H2O/D2O water mixture (blue line) matches the SLD of the biomolecule. Figure was adapted from (Jacrot 1976). 


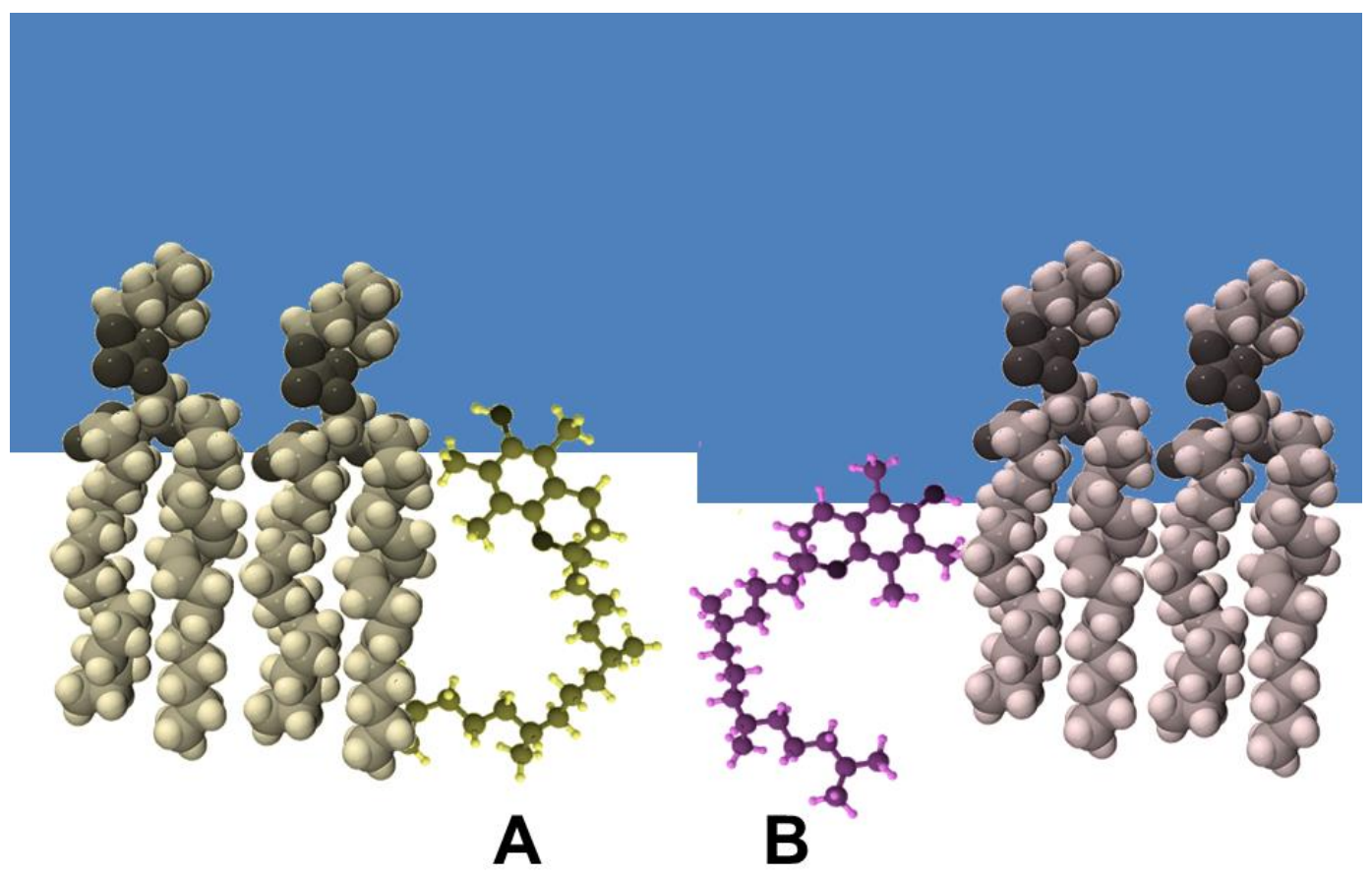

Figure 3 Schematic of the location of alpha-tocopherol in model membranes. $A$, In lipid bilayers composed of 1,2-dioleoyl-sn-glycero-3-phosphocholine (DOPC), POPC or 1-palmitoyl-2arachidonoyl-sn-glycero-3-phosphocholine, $\alpha$-tocopherol resides further away from the bilayer center compared to bilayers $(B)$ composed of DPPC, POPC or 1,2-arachidonoyl-sn-glycero-3phosphocholine. Figure adapted from (Marquardt, Williams et al. 2013). 


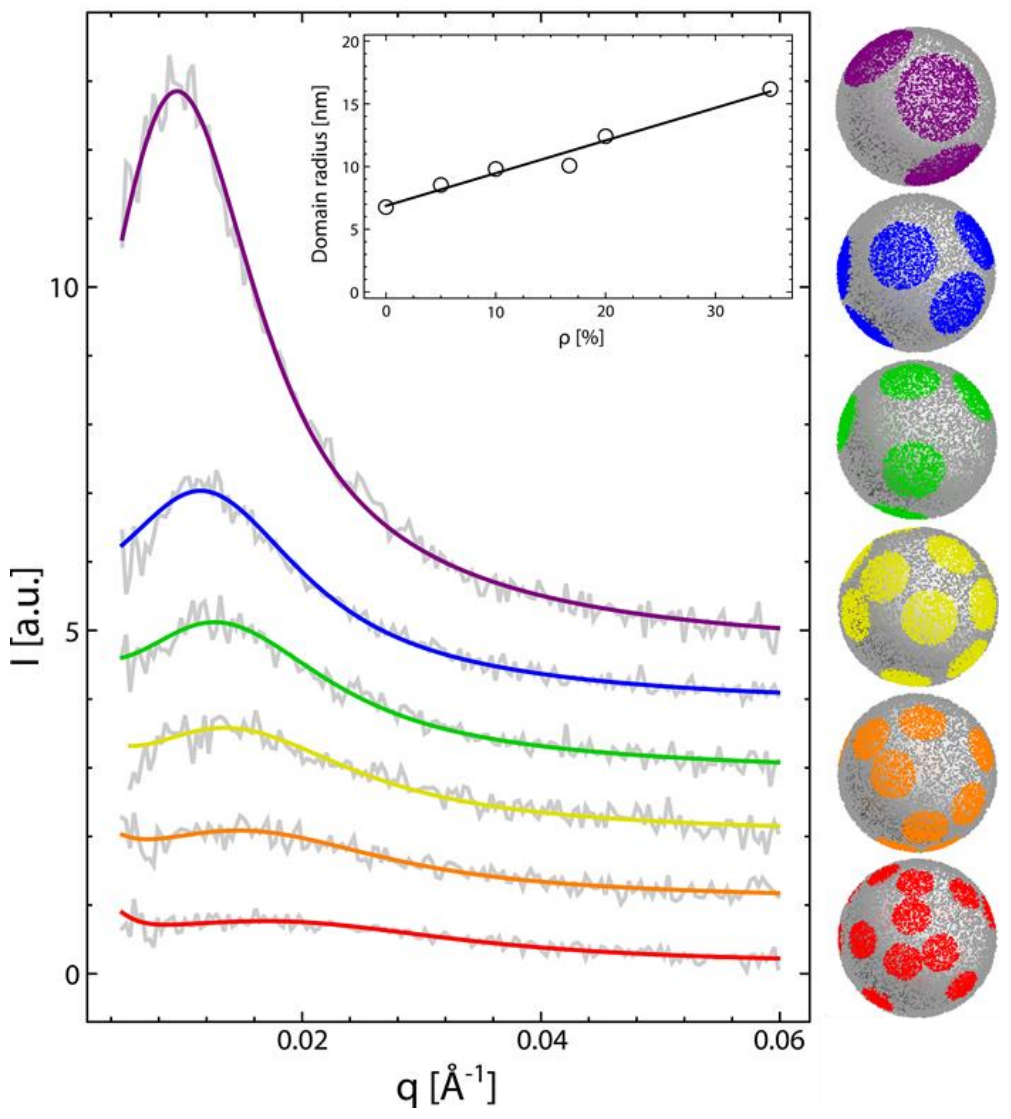

Figure 4 Small-angle neutron scattering from contrast matched $60 \mathrm{~nm}$ diameter vesicles reveals a domain size transition driven by lipid composition. Scattered intensity from vesicle suspensions (light gray lines) were fit with Monte Carlo simulations (colored lines) of spherical shells containing monodisperse domains of variable size and number. The inset shows the best fit domain radius $v s$. the ratio of $\mathrm{DOPC} /(\mathrm{DOPC}+\mathrm{POPC})$ in the lipid mixture. Example Monte Carlo vesicles corresponding to the best fit parameters are shown on the right. Figure adapted from (Heberle, Petruzielo et al. 2013). 


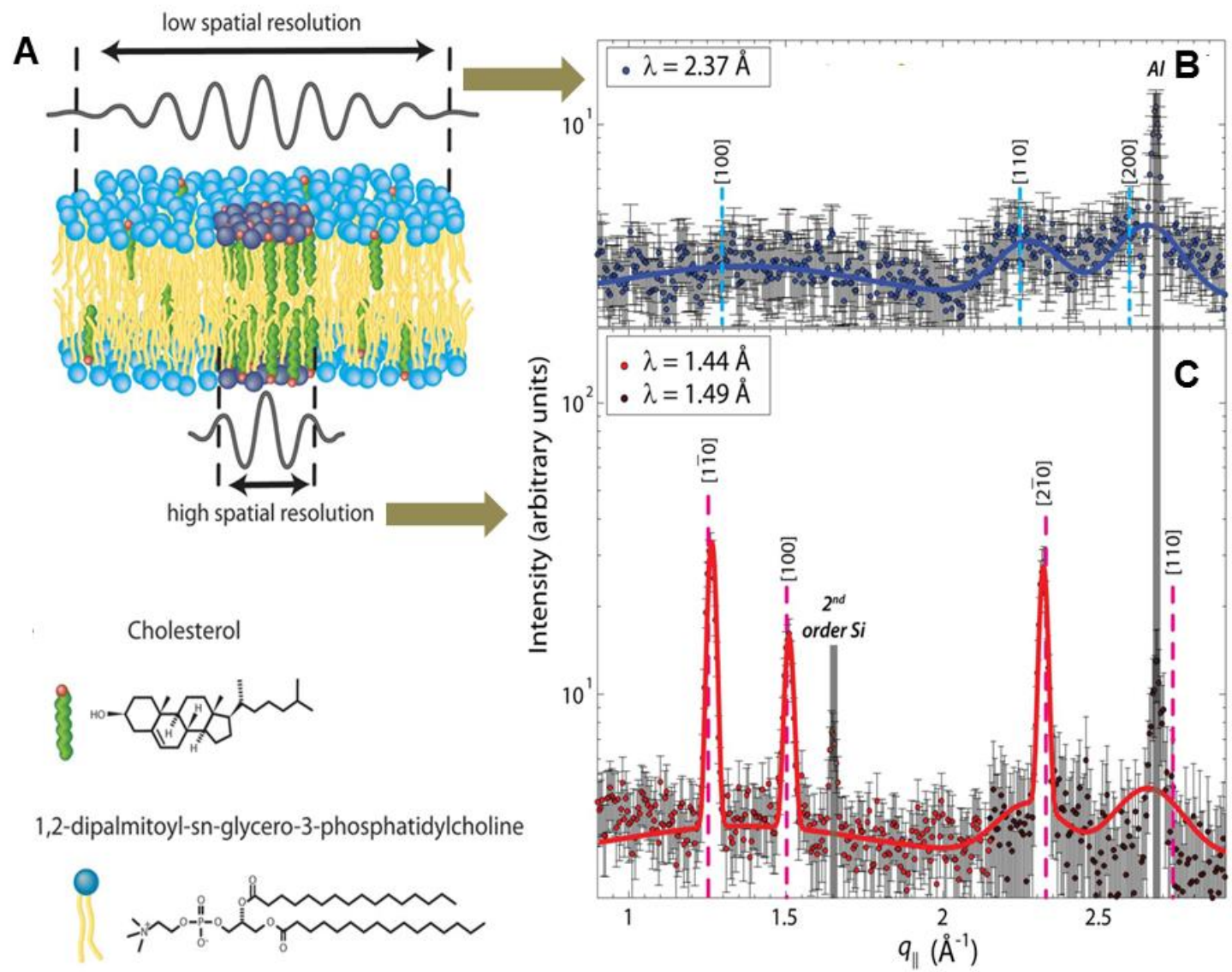

Figure 5 Neutron coherence length variation reveals bilayer substructure. $A$, Schematic of a lipid bilayer with cholesterol rich nanoscopic domains. The low and high spatial resolution neutron scattering setups result in the diffraction patterns in $B$ and $C$, respectively. The neutron coherence length was varied from $\sim 91 \AA(B)$ to $\sim 32 \AA(C)$. When the neutron coherence length becomes comparable in size to the nanoscopic domain, structural features become better resolved, as indicated by the sharper Bragg reflections in $C$ compared to $B$. Figure adapted from (Armstrong, Marquardt et al. 2013). 


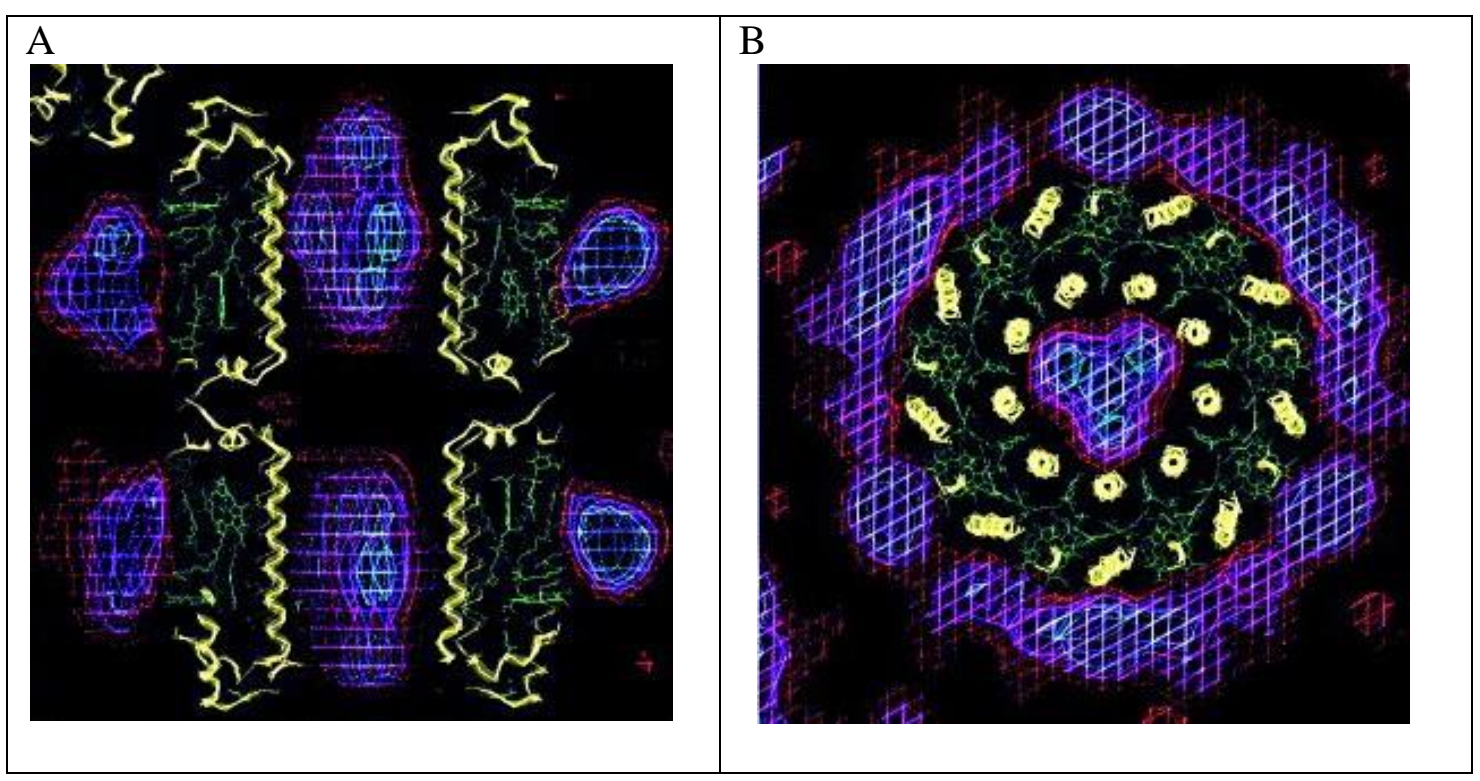

Figure 6 Neutron diffraction with contrast variation resolves the location of H/D-labeled detergent tails groups in the single crystal structure of the LH2 light harvesting complex: the red contours enclose detergent tail volumes of $16 \%$, magenta $8 \%$, and cyan $2.5 \%$ of the cell volume. Protein is shown as yellow ribbons and pigment molecules in green. A) Side elevation showing a cross section through the membrane spanning annular LH2 complex, and B) plan view looking down on the LH2 complex. Figure adapted from (Prince et al, 2003) 


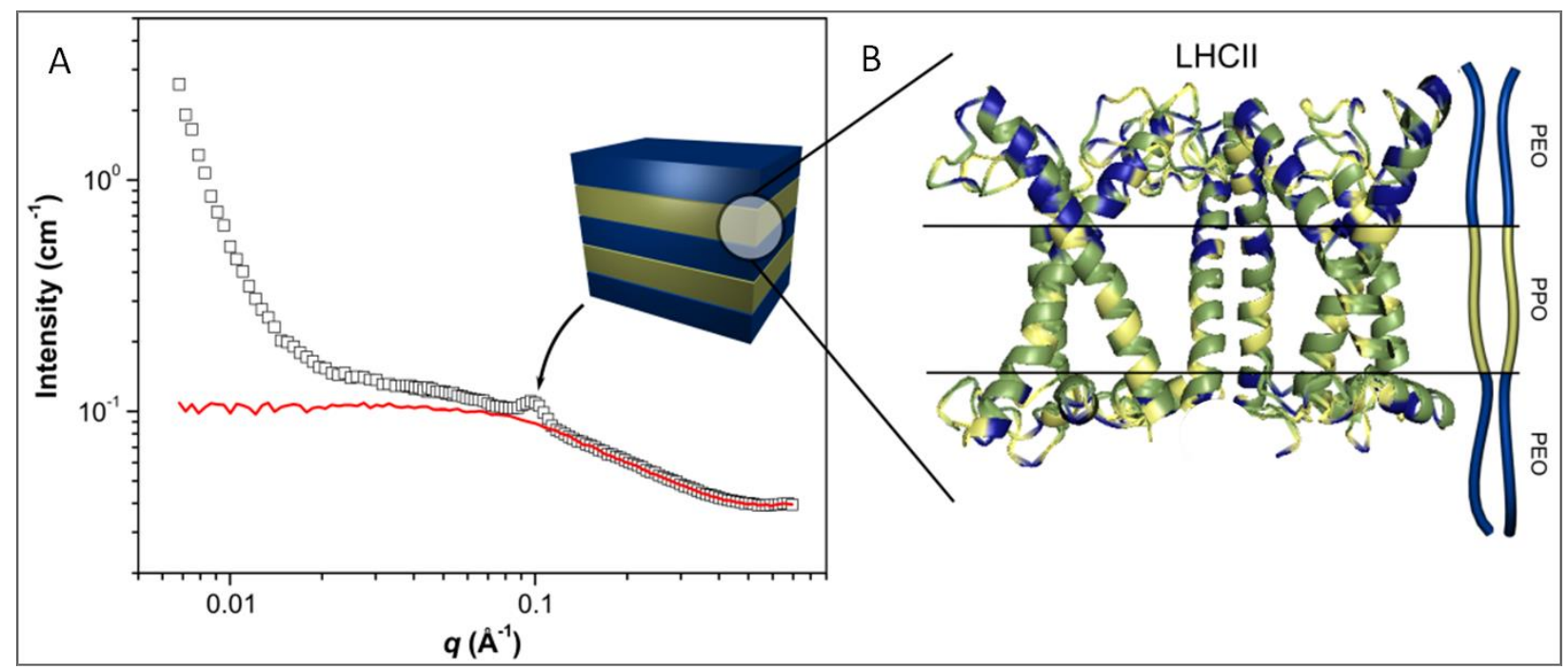

Figure 7 Schematic representation showing a photoactive biohybrid membrane formed by the self-assembly of naturally occurring light harvesting antennae with synthetic block copolymers. (A) The SANS profile of LH2 light harvesting complex in $20 \%$ (w/w) $\mathrm{PEO}_{43}-\mathrm{PPO}_{16}-\mathrm{PEO}_{43}$ (squares) and the solid line is the SANS profile of $\mathrm{PEO}_{43}-\mathrm{PPO}_{16}-\mathrm{PEO}_{43}$ in detergent solution. (B) Schematic representation of the interaction of $\mathrm{PEO}_{43}-\mathrm{PPO}_{16}-\mathrm{PEO}_{43}$ and $\mathrm{LH} 2$ in lamellar environment. The blue, yellow, and green colors of the polypeptide backbone represent polar charged, polar uncharged, and hydrophobic amino acid residues, respectively. Figure adapted from (Cardoso et al., 2011). 


\section{REFERENCES}

Ankner, J. F., W. T. Heller, et al. (2013). "Neutron scattering techniques and applications in structural biology." Curr Protoc Protein Sci Chapter 17: Unit17 16.

Armstrong, C. L., M. A. Barrett, et al. (2012). "Co-existence of gel and fluid lipid domains in single-component phospholipid membranes." Soft Matter 8(17): 4687-4694.

Armstrong, C. L., D. Marquardt, et al. (2013). "The Observation of Highly Ordered Domains in Membranes with Cholesterol." Plos One 8(6).

Atkinson, J., R. F. Epand, et al. (2008). "Tocopherols and tocotrienols in membranes: A critical review." Free Radical Biology and Medicine 44(5): 739-764.

Azzi, A. (2007). "Molecular mechanism of alpha-tocopherol action." Free Radical Biology and Medicine 43(1): 16-21.

Bramkamp, M. and D. Lopez (2015). "Exploring the existence of lipid rafts in bacteria." Microbiol Mol Biol Rev 79(1): 81-100.

Breyton, C., F. Gabel, et al. (2013). "Small angle neutron scattering for the study of solubilised membrane proteins." Eur. Phys. J. E - Soft Matter 36(7): 71(71)-71(16).

Buldt, G., H. U. Gally, et al. (1978). "Neutron-Diffraction Studies on Selectively Deuterated Phospholipid Bilayers." Nature 271(5641): 182-184.

Buldt, G., H. U. Gally, et al. (1979). "Neutron-Diffraction Studies on Phosphatidylcholine Model Membranes .1. Head Group Conformation." J. Mol. Biol. 134(4): 673-691.

Burge, B. W. and A. S. Huang (1970). "Comparison of membrane protein glycopeptides of Sindbis virus and vesicular stomatitis virus." Journal of virology 6(2): 176-182.

Burgess, I., M. Li, et al. (2004). "Electric field-driven transformations of a supported model biological membrane - An electrochemical and neutron reflectivity study." Biophysical Journal 86(3): 1763-1776.

Cardoso, M. B., D. Smolensky, et al. (2011). "Supramolecular assembly of biohybrid photoconversion systems." Energy \& Environmental Science 4(1): 181-188.

Cardoso, M. B., D. Smolensky, et al. (2009). "Insight into the structure of light-harvesting complex II and its stabilization in detergent solution." J Phys Chem B 113(51): 1637716383.

Chadwick, J. (1932). "The possible existence of a neutron." Nature 129: 312.

Chaudhuri, B. N., S. Gupta, et al. (2011). "A combined global and local approach to elucidate spatial organization of the Mycobacterial ParB-parS partition assembly." Biochemistry 50(11): 1799-1807.

Clifton, L. A., C. L. Johnson, et al. (2012). "Low resolution structure and dynamics of a colicinreceptor complex determined by neutron scattering." J Biol Chem 287(1): 337-346.

Deme, B., C. Cataye, et al. (2014). "Contribution of galactoglycerolipids to the 3-dimensional architecture of thylakoids." Faseb Journal 28(8): 3373-3383.

Doshi, D. A., A. M. Dattelbaum, et al. (2005). "Neutron reflectivity study of lipid membranes assembled on ordered nanocomposite and nanoporous silica thin films." Langmuir 21(7): 2865-2870.

Emerson, O. H., G. A. Emerson, et al. (1936). "The isolation from cottonseed oil of an alcohol resembling a-tocopherol from wheat-germ oil " Science 83: 421.

Evans, H. M. and K. S. Bishop (1922). "On the existence of a hitherto unrecognized dietary factor essential for reproduction." Science 56(1458): 650-651. 
Evans, H. M., O. H. Emerson, et al. (1936). "The isolation from wheat germ oil of an alcohol, atocopherol, having the properties of vitamin E " J. Biol. Chem. 113: 319-332.

Fitzsimmons, M. R., S. D. Bader, et al. (2004). "Neutron scattering studies of nanomagnetism and artificially structured materials." J Mag. Mag. Mater. 271(1): 103-146.

Fragneto, G., F. Graner, et al. (2000). "Interaction of the third helix of Antennapedia homeodomain with a deposited phospholipid bilayer: A neutron reflectivity structural study." Langmuir 16(10): 4581-4588.

Gajate, C., F. Gonzalez-Camacho, et al. (2009). "Lipid raft connection between extrinsic and intrinsic apoptotic pathways." Biochem Biophys Res Commun 380(4): 780-784.

Garman, E. F. (2014). "Developments in X-ray crystallographic structure determination of biological macromolecules." Science 343: 1102-1108.

Gerbeau-Pissot, P., C. Der, et al. (2014). "Modification of plasma membrane organization in tobacco cells elicited by cryptogein." Plant Physiol 164(1): 273-286.

Golden, E. A. and A. Vrielink (2014). "Looking for Hydrogen Atoms: Neutron Crystallography Provides Novel Insights Into Protein Structure and Function." Australian Journal of Chemistry 67(12): 1751-1762.

Halban, H. v. and P. Preiswerk (1936). "Preuve expérimentale de la diffraction des neutrons." $\underline{\text {. }}$ R. Acad. Sci. Paris 203: 73-75.

Han, X., K. Hristova, et al. (2008). "Protein folding in membranes: Insights from neutron diffraction studies of a membrane beta-sheet oligomer." Biophys. J. 94(2): 492-505.

Harroun, T. A., N. Kucerka, et al. (2009). "Neutron and X-ray scattering for biophysics and biotechnology: examples of self-assembled lipid systems." Soft Matter 5(14): 2694-2703.

He, L., A. Piper, et al. (2012). "Conformational changes in Sindbis virus induced by decreased pH are revealed by small-angle neutron scattering." J Virol 86(4): 1982-1987.

He, L., A. Piper, et al. (2010). "The structure of Sindbis virus produced from vertebrate and invertebrate hosts as determined by small-angle neutron scattering." J Virol 84(10): 52705276.

Head, B. P., H. H. Patel, et al. (2014). "Interaction of membrane/lipid rafts with the cytoskeleton: impact on signaling and function: membrane/lipid rafts, mediators of cytoskeletal arrangement and cell signaling." Biochim Biophys Acta 1838(2): 532-545.

Heberle, F. A., M. Doktorova, et al. (2013). "Hybrid and nonhybrid lipids exert common effects on membrane raft size and morphology." J Am Chem Soc 135(40): 14932-14935.

Heberle, F. A., J. Pan, et al. (2012). "Model-based approaches for the determination of lipid bilayer structure from small-angle neutron and X-ray scattering data." Eur Biophys J 41(10): 875-890.

Heberle, F. A., R. S. Petruzielo, et al. (2013). "Bilayer thickness mismatch controls domain size in model membranes." J. Am. Chem. Soc. 135: 6853-6859.

Heftberger, P., B. Kollmitzer, et al. (2014). "Global small-angle X-ray scattering data analysis for multilamellar vesicles: the evolution of the scattering density profile model." $\mathrm{J}$ Appl Crystallogr 47(Pt 1): 173-180.

Heinrich, F. and M. Losche (2014). "Zooming in on disordered systems: neutron reflection studies of proteins associated with fluid membranes." Biochim Biophys Acta 1838(9): 2341-2349.

Heller, W. T. (2010). "Small-angle neutron scattering and contrast variation: a powerful combination for studying biological structures." Acta Crystallogr D Biol Crystallogr 66(Pt 11): 1213-1217. 
Hellstrand, E., M. Grey, et al. (2013). "Adsorption of alpha-synuclein to supported lipid bilayers: positioning and role of electrostatics." ACS Chem Neurosci 4(10): 1339-1351.

Hertrich, S., F. Stetter, et al. (2014). "Highly hydrated deformable polyethylene glycol-tethered lipid bilayers." Langmuir 30(31): 9442-9447.

Holowka, D., J. A. Gosse, et al. (2005). "Lipid segregation and IgE receptor signaling: a decade of progress." Biochim Biophys Acta 1746(3): 252-259.

Holt, S. A., A. P. Le Brun, et al. (2009). "An ion-channel-containing model membrane: structural determination by magnetic contrast neutron reflectometry." Soft Matter 5(13): 25762586.

Hughes, A. V., J. R. Howse, et al. (2008). "Floating lipid bilayers deposited on chemically grafted phosphatidylcholine surfaces." Langmuir 24(5): 1989-1999.

Jacobson, K., O. G. Mouritsen, et al. (2007). "Lipid rafts: at a crossroad between cell biology and physics." Nature Cell Biology 9(1): 7-14.

Jacrot, B. (1976). "Study of Biological Structures by Neutron-Scattering from Solution." Reports on Progress in Physics 39(10): 911-953.

Jayasundar, J. J., J. H. Ju, et al. (2012). "Open conformation of ezrin bound to phosphatidylinositol 4,5-bisphosphate and to F-actin revealed by neutron scattering." $\underline{\mathbf{J}}$ Biol Chem 287(44): 37119-37133.

Johs, A., M. Hammel, et al. (2006). "Modular structure of solubilized human apolipoprotein B100. Low resolution model revealed by small angle neutron scattering." J Biol Chem 281(28): 19732-19739.

Karrer, P., H. Fritzsche, et al. (1938). "Synthesis of a-Tocopherol (vitamin E)." Nature 141(3580): 1057.

Karrer, P., H. Fritzsche, et al. (1938). "a-Tocopherol." Helv. Chim. Acta 21(1): 520-525.

Katsaras, J. (1997). "Highly aligned lipid membrane systems in the physiologically relevant "excess water" condition." Biophys. J. 73(6): 2924-2929.

Katsaras, J. (1998). "Adsorbed to a rigid substrate, dimyristoylphosphatidylcholine multibilayers attain full hydration in all mesophases." Biophys. J. 75(5): 2157-2162.

Kent, B., T. Hunt, et al. (2014). "Localization of trehalose in partially hydrated DOPC bilayers: insights into cryoprotective mechanisms." Journal of the Royal Society Interface 11(95).

Kent, M. S., J. K. Murton, et al. (2010). "Neutron Reflectometry Study of the Conformation of HIV Nef Bound to Lipid Membranes." Biophysical Journal 99(6): 1940-1948.

Kent, M. S., H. Yim, et al. (2008). "Oligomerization of membrane-bound diphtheria toxin (CRM197) facilitates a transition to the open form and deep insertion." Biophysical Journal 94(6): 2115-2127.

King, G. I. and S. H. White (1986). "Determining bilayer hydrocarbon thickness from neutron diffraction measurements using strip-function models." Biophys J 49(5): 1047-1054.

Knight, R. L., K. L. W. Schultz, et al. (2009). Role of N-Linked Glycosylation for Sindbis Virus Infection and Replication in Vertebrate and Invertebrate Systems. 83: 5640-5647.

Krueger, S., J. F. Ankner, et al. (1995). "Extending the Angular Range of Neutron Reflectivity Measurements from Planar Lipid Bilayers - Application to a Model Biological Membrane." Langmuir 11(8): 3218-3222.

Krueger, S., C. W. Meuse, et al. (2001). "Investigation of hybrid bilayer membranes with neutron reflectometry: Probing the interactions of melittin." Langmuir 17(2): 511-521.

Kucerka, N., J. Gallova, et al. (2009). "Areas of monounsaturated diacylphosphatidylcholines." Biophys J 97(7): 1926-1932. 
Kucerka, N., D. Marquardt, et al. (2010). "Cholesterol in Bilayers with PUFA Chains: Doping with DMPC or POPC Results in Sterol Reorientation and Membrane-Domain Formation." Biochemistry 49(35): 7485-7493.

Kucerka, N., J. F. Nagle, et al. (2008). "Lipid bilayer structure determined by the simultaneous analysis of neutron and X-ray scattering data." Biophys J 95(5): 2356-2367.

Kucerka, N., M. P. Nieh, et al. (2011). "Fluid phase lipid areas and bilayer thicknesses of commonly used phosphatidylcholines as a function of temperature." Biochim Biophys Acta 1808(11): 2761-2771.

Kucerka, N., B. van Oosten, et al. (2015). "Molecular structures of fluid phosphatidylethanolamine bilayers obtained from simulation-to-experiment comparisons and experimental scattering density profiles." J Phys Chem B 119(5): 1947-1956.

Kusaka, K., T. Hosoya, et al. (2013). "Evaluation of performance for IBARAKI biological crystal diffractometer iBIX with new detectors." J Synchrotron Radiat 20(Pt 6): 994-998.

Le Brun, A. P., S. A. Holt, et al. (2008). "Monitoring the assembly of antibody-binding membrane protein arrays using polarised neutron reflection." European Biophysics Journal with Biophysics Letters 37(5): 639-645.

Le, R. K., B. J. Harris, et al. (2014). "Analysis of the solution structure of Thermosynechococcus elongatus photosystem I in n-dodecyl-beta-D-maltoside using small-angle neutron scattering and molecular dynamics simulation." Archives of Biochemistry and Biophysics 550: 50-57.

Liberton, M., A. M. Collins, et al. (2013). "Probing the consequences of antenna modification in cyanobacteria." Photosynthesis Research 118(1-2): 17-24.

Liberton, M., L. E. Page, et al. (2013). "Organization and Flexibility of Cyanobacterial Thylakoid Membranes Examined by Neutron Scattering." J. Biol. Chem. 288(5): 36323640 .

Liberton, M., L. E. Page, et al. (2013). "Organization and Flexibility of Cyanobacterial Thylakoid Membranes Examined by Neutron Scattering." Journal of Biological Chemistry 288(5): 3632-3640.

Lingwood, D. and K. Simons (2010). "Lipid Rafts As a Membrane-Organizing Principle." Science 327(5961): 46-50.

Marquardt, D., J. A. Williams, et al. (2013). "Tocopherol activity correlates with its location in a membrane:a new perspective on the antioxidant vitamin E." J. Am. Chem. Soc. 135: 7523-7533.

Martin, S. L., L. He, et al. (2013). "New insight into the structure of RNA in red clover necrotic mosaic virus and the role of divalent cations revealed by small-angle neutron scattering." Arch Virol 158: 1661-1669.

Mason, T. E., T. J. Gawne, et al. (2013). "The early development of neutron diffraction: science in the wings of the Manhattan Project." Acta Crystallographica Section A 69: 37-44.

Mehan, S., A. J. Chinchalikar, et al. (2013). "Small-angle neutron scattering study of structure and interaction of nanoparticle, protein and surfactant complexes." Langmuir 29(36): 11290-11299.

Meilleur, F., P. Munshi, et al. (2013). "The IMAGINE instrument: first neutron protein structure and new capabilities for neutron macromolecular crystallography." Acta Crystallogr D Biol Crystallogr 69(Pt 10): 2157-2160.

Midtgaard, S. R., M. C. Pedersen, et al. (2014). "Self-assembling peptides form nanodiscs that stabilize membrane proteins." Soft Matter 10(5): 738-752. 
Mihailescu, M., D. Krepkiy, et al. (2014). "Structural interactions of a voltage sensor toxin with lipid membranes." Proc Natl Acad Sci U S A 111(50): E5463-5470.

Mihailescu, M., R. G. Vaswani, et al. (2011). "Acyl-Chain Methyl Distributions of LiquidOrdered and -Disordered Membranes." Biophysical Journal 100(6): 1455-1462.

Mitchell, D. P. and P. N. Powers (1936). "Bragg reflection of slow neutrons." Phys. Rev. 50: 486-487.

Morales-Penningston, N. F., J. Wu, et al. (2010). "GUV preparation and imaging: minimizing artifacts." Biochim. Biophys. Acta - Biomembranes 1798(7): 1324-1332.

Murai, T. (2012). "The role of lipid rafts in cancer cell adhesion and migration." Int J Cell Biol 2012: 763283.

Nagle, J. F. and S. Tristram-Nagle (2000). "Structure of lipid bilayers." Biochim Biophys Acta 1469(3): 159-195.

Nanda, H., S. A. K. Datta, et al. (2010). "Electrostatic Interactions and Binding Orientation of HIV-1 Matrix Studied by Neutron Reflectivity." Biophysical Journal 99(8): 2516-2524.

O'Dell, W. B., K. J. Beatty, et al. (2012). "Sol-gel entrapped light harvesting antennas: immobilization and stabilization of chlorosomes for energy harvesting." Journal of Materials Chemistry 22(42): 22582-22591.

Ono, A. (2010). "Relationships between plasma membrane microdomains and HIV-1 assembly." Biol Cell 102(6): 335-350.

Pabst, G., N. Kučerka, et al. (2010). "Applications of neutron and x-ray scattering to the syudy of biologically relevant model membranes." Chem. Phys. Lipids 163: 460-479.

Pan, J., X. Cheng, et al. (2012). "Interactions between ether phospholipids and cholesterol as determined by scattering and molecular dynamics simulations." J. Phys. Chem. B 116: 14829-14838.

Pan, J., X. Cheng, et al. (2014). "The molecular structure of a phosphatidylserine bilayer determined by scattering and molecular dynamics simulations." Soft Matter 10: 37163725.

Pan, J., X. Cheng, et al. (2015). "Structural and mechanical properties of cardiolipin lipid bilayers determined using neutron spin echo, small angle neutron and X-ray scattering, and molecular dynamics simulations." Soft Matter 11(1): 130-138.

Pan, J., F. A. Heberle, et al. (2013). "Using small-angle neutron scattering to detect nanoscopic lipid domains." Chem Phys Lipids 170-171: 19-32.

Pan, J., D. Marquardt, et al. (2014). "Revisiting the bilayer structures of fluid phase phosphatidylglycerol lipids: Accounting for exchangeable hydrogens." Biochim Biophys Acta 1838(11): 2966-2969.

Penel, S., E. Pebay-Peyroula, et al. (1998). "Detergent binding in trigonal crystals of OmpF porin from Escherichia coli." Biochimie 80(5-6): 543-551.

Perevozchikova, T., C. B. Stanley, et al. (2014). "Investigating the structural impact of the glutamine repeat in huntingtin assembly." Biophys J 107(2): 411-421.

Petruzielo, R. S., F. A. Heberle, et al. (2013). "Phase behavior and domain size in sphingomyelin-containing lipid bilayers." Biochim Biophys Acta 1828(4): 1302-1313.

Prince, S. M., T. D. Howard, et al. (2003). "Detergent structure in crystals of the integral membrane light-harvesting complex LH2 from Rhodopseudomonas acidophila strain 10050." J Mol Biol 326(1): 307-315.

Qian, S., R. Dean, et al. (2012). "The internal organization of mycobacterial partition assembly: does the DNA wrap a protein core?" PLoS One 7(12): e52690. 
Roth, M., A. Lewitbentley, et al. (1989). "Detergent Structure in Crystals of a Bacterial Photosynthetic Reaction Center." Nature 340(6235): 659-662.

Schultz, A. J., P. Thiyagarajan, et al. (2005). "Conceptual design of a macromolecular neutron diffractometer (MaNDi) for the SNS." Journal of Applied Crystallography 38: 964-974.

Sears, V. F. (1992). "Neutron scattering lengths and cross sections." Neutron News 3: 26-37.

Sebastiao, A. M., M. Colino-Oliveira, et al. (2013). "Lipid rafts, synaptic transmission and plasticity: impact in age-related neurodegenerative diseases." Neuropharmacology 64: 97-107.

Shen, H. H., D. L. Leyton, et al. (2014). "Reconstitution of a nanomachine driving the assembly of proteins into bacterial outer membranes." Nat Commun 5: 5078.

Shin, E., S.-H. Choi, et al. (2010). "Small-angle neutron scattering study on the cold rolled steel sheets." Appl. Phys. A 99: 621-625.

Simons, K. and M. J. Gerl (2010). "Revitalizing membrane rafts: new tools and insights." Nat Rev Mol Cell Biol 11(10): 688-699.

Stidder, B., G. Fragneto, et al. (2005). "Cholesterol induced suppression of large swelling of water layer in phosphocholine floating bilayers." Langmuir 21(19): 8703-8710.

Strandberg, B., R. E. Dickerson, et al. (2009). "50 years of protein structure analysis." J. Mol. Biol. 392: 2-32.

Tanaka, I., K. Kusaka, et al. (2010). "Neutron structure analysis using the IBARAKI biological crystal diffractometer (iBIX) at J-PARC." Acta Crystallogr D Biol Crystallogr 66(Pt 11): 1194-1197.

Tang, K. H. and R. E. Blankenship (2012). "Neutron and light scattering studies of lightharvesting photosynthetic antenna complexes." Photosynth Res 111(1-2): 205-217.

Tang, K. H., V. S. Urban, et al. (2010). "SANS investigation of the photosynthetic machinery of Chloroflexus aurantiacus." Biophys J 99(8): 2398-2407.

Tang, K. H., L. Zhu, et al. (2011). "Temperature and ionic strength effects on the chlorosome light-harvesting antenna complex." Langmuir 27(8): 4816-4828.

Tronin, A. Y., C. E. Nordgren, et al. (2014). "Direct evidence of conformational changes associated with voltage gating in a voltage sensor protein by time-resolved X-ray/neutron interferometry." Langmuir 30(16): 4784-4796.

Vacklin, H. P., F. Tiberg, et al. (2005). "Phospholipase A(2) hydrolysis of supported phospholipid bilayers: A neutron reflectivity and ellipsornetry study." Biochemistry 44(8): 2811-2821.

Wiener, M. C., G. I. King, et al. (1991). "Structure of a fluid dioleoylphosphatidylcholine bilayer determined by joint refinement of x-ray and neutron diffraction data. I. Scaling of neutron data and the distributions of double bonds and water." Biophys J 60(3): 568-576.

Wiener, M. C. and S. H. White (1991). "Fluid bilayer structure determination by the combined use of x-ray and neutron diffraction. I. Fluid bilayer models and the limits of resolution." Biophys J 59(1): 162-173.

Wiener, M. C. and S. H. White (1991). "Fluid bilayer structure determination by the combined use of x-ray and neutron diffraction. II. "Composition-space" refinement method." Biophys J 59(1): 174-185.

Wiener, M. C. and S. H. White (1992). "Structure of a fluid dioleoylphosphatidylcholine bilayer determined by joint refinement of x-ray and neutron diffraction data. II. Distribution and packing of terminal methyl groups." Biophys J 61(2): 428-433. 
Wiener, M. C. and S. H. White (1992). "Structure of a fluid dioleoylphosphatidylcholine bilayer determined by joint refinement of $x$-ray and neutron diffraction data. III. Complete structure." Biophys J 61(2): 434-447.

Wollan, E. O. and C. G. Shull (1948). "The Diffraction of Neutrons by Crystalline Powders." Phys. Rev. 73(8): 830-841.

Worcester, D. L. and N. P. Franks (1976). "Structural-Analysis of Hydrated Egg Lecithin and Cholesterol Bilayers .2. Neutron-Diffraction." J. Mol. Biol. 100(3): 359-378.

Zaccai, G., G. Buldt, et al. (1979). "Neutron-Diffraction Studies on Phosphatidylcholine Model Membranes .2. Chain Conformation and Segmental Disorder." J. Mol. Biol. 134(4): 693-706.

Zinn, W. H. (1947). "Diffraction of Neutrons by a Single Crystal." Phys. Rev. 71(11): 752-757. 